\title{
Welche Maske bei Schweinegrippe?
}

\author{
Roland Schulze-Röbbecke
}

Mit der neuerlichen Diskussion über eine bevorstehende Influenza-Pandemie wird wieder die Frage nach dem Schutzeffekt diverser Masken gestellt. Bei der Wahl solcher Maßnahmen ist bekanntlich der Übertragungsweg zu berücksichtigen, wobei prinzipiell zwischen Kontakt-, Tröpfchen- und aerogener Übertragung unterschieden wird [1,2]. Zum Schutz vor einer Kontaktübertragung stehen Maßnahmen wie die Händedesinfektion im Vordergrund. Vor einer Tröpfchenübertragung schützen normale OP-Masken (= Mund-Nasen-Schutz), während vor einer aerogenen Übertragung kostspielige Atemschutzmasken (z.B. FFP2) und die Unterbringung infektiöser Patienten in Räumen mit Luftunterdruck schützen.

Über die Frage, wie die Influenza - und besonders die „Schweinegrippe“ - übertragen wird, herrscht keine Einigkeit. Als Hauptübertragungsweg der saisonalen Influenza gelten die Tröpfchenübertragung über kurze Distanzen und die Kontaktübertragung [2-4]. Weitgehend unbekannt ist das Risiko der aerogenen Influenzaübertragung, für die es nur vage Hinweise gibt [2,3], die allerdings unterschiedlich interpretiert werden:

- Die Weltgesundheitsorganisation (WHO) geht unter normalen Bedingungen von der Tröpfchenübertragung über eine Distanz von maximal $1 \mathrm{~m}$ aus und empfiehlt bei engerem Patientenkontakt das Tragen von OP-Masken [5]. Nur bei Aerosol-generierenden Tätigkeiten wie der Bronchoskopie empfiehlt die WHO FFP2-Atemschutzmasken.

- Die US-amerikanischen Centers for Disease Control and Prevention (CDC) empfehlen bei Betreten des Zimmers eines „Schweinegrippe“-Patienten dagegen eine N95- (entsprechend FFP2-) Atemschutzmaske, da über das neue H1N1-Virus noch nicht genug bekannt sei [6]. Für Aerosol-generierende Tätigkeiten wird ein belüfteter Raum mit Luftunterdruck empfohlen.

- Der deutsche Ausschuss für Biologische Arbeitsstoffe (ABAS) geht offenbar von der aerogenen Übertragung der nicht impfpräventablen Influenza aus und gibt komplizierte Empfehlungen zum Gebrauch von FFP1-, FFP2- und FFP3-Atemschutzmasken, je nach Ausmaß der Aerosolbildung [7]. Auf die Raumlüftung wird nicht eingegangen, obwohl diese beim Schutz vor aerogen übertragenen Infektionen berücksichtigt werden sollte [2].

Solch unterschiedliche Empfehlungen verwirren, spiegeln aber unser lückenhaftes Wissen wider. Keine Maßnahme wird einen absolut sicheren Schutz garantieren können, und es stellt sich die Frage, ob das Kompliziertere und Teurere immer auch das Bessere ist.

\section{Literatur}

1 Schulze-Röbbecke R. Isolierung infektiöser Patienten - auf die Übertragungswege kommt es an. Krankenhaushygiene up2date 2006; 1: 97 - 114

2 Siegel JD, Rhinehart E, Jackson M et al. Guideline for isolation precautions: preventing transmission of infectious agents in healthcare settings. http:// www.cdc.gov/ncidod/dhqp/pdf/guidelines/Isolation2007.pdf (Stand: Juni 2007)

3 Bridges CB, Kuehnert MJ, Hall CB. Transmission of influenza: implications for control in health care settings. Clin Infect Dis 2003; 37: $1094-1101$

4 Brankston G, Gitterman L, Hirji Z et al. Transmission of influenza $A$ in human beings. Lancet Infect Dis 2007; 7: $257-265$

5 World Health Organization. Infection prevention and control in health care in providing care for confirmed or suspected A (H1N1) swine influenza patients. http://www.who.int/csr/resources/publications/20 090429_infection_control_en.pdf (Stand: 29. 4. 2009)

6 Centers for Disease Control and Prevention. Interim guidance for infection control for care of patients with confirmed or suspected novel influenza $A$ (H1N1) virus infection in a healthcare setting. http://www.cdc.gov/h1n1flu/guidelines_infection_control.htm (Stand: 13. 5. 2009)

7 AusschussfürBiologischeArbeitsstoffe. Beschluss 609, Arbeitsschutz beim Auftreten von nicht impfpräventabler Influenza unter besonderer Berücksichtigung des Arbeitsschutzes. http://www.baua. de/nn_15408/de/Themen-von-A-Z/ Biologische-Arbeitsstoffe/TRBA/pdf/Beschluss-609. pdf (Ausgabe Dezember 2006, letzter Zugriff: 31. 5. 2009) 